\title{
Rotor Position Detection of CPPM Belt Starter Generator with Trapezoidal Back EMF using Six Hall Sensors
}

\author{
Xu Jiaqun*, Long Feng, and Cui Haotian \\ College of Electronic Information and Control Engineering, Beijing University of Technology, Beijing 100124, China
}

(Received 25 December 2015, Received in final form 16 March 2016, Accepted 17 March 2016)

\begin{abstract}
Six-step commutation control widely used in brushless DC (BLDC) motor can be applied to consequent pole permanent magnet (CPPM) belt starter generator (BSG) with trapezoidal back electromotive force (EMF) in the starter state. However, rotor position detection with three Hall sensors in BLDC motor can hardly be employed in CPPM BSG due to asymmetric flux distribution in each pole side of CPPM BSG. This paper presents a low-cost rotor position detection method for CPPM BSG in which six Hall sensors are proposed to be used based on the analysis of flux distribution by 3D FEA. In the method, the six Hall sensors are divided into three groups and two signals in each group are combined through performing logic operations. In addition, offset angle between back EMF and the related Hall signal can be compensated by moving the Hall sensors. Experiments of a $2 \mathrm{~kW}$ CPPM BSG prototype have also been performed to verify the proposed method.
\end{abstract}

Keywords : Rotor position detection, consequent pole permanent magnet (CPPM), belt starter generator (BSG), Hall sensor

\section{Introduction}

With the development of electric machines and power electronics technologies, belt starter generator (BSG), which is connected to the internal combustion engine through a belt, has become attractive for automobiles. BSG has two operating states, one offering constant voltage in wide speed range as a generator and the other offering maximum torque output for quick engine cranking as a starter [1]. As a hybrid excitation machine, consequent pole permanent magnet (CPPM) machine is a potential candidate for BSG applications with the advantages of high torque capability, high power density and controllable air gap flux $[2,3]$.

In the starter state, the maximum field current is critical for offering enough torque for quick engine cranking, and CPPM BSG with trapezoidal back electromotive force (EMF) can be regarded as a brushless DC (BLDC) motor in view of the same back EMF waveform. In addition, six-step commutation control widely used in BLDC motor can be adopted [4] and rotor position is required for synchronizing the current direction with the back

(C)The Korean Magnetics Society. All rights reserved.

*Corresponding author: Tel: +010-67392391

Fax: +010-67391625, e-mail: xjq@bjut.edu.cn
EMF direction. In the generator state, uncontrolled diode rectifier can be used and rotor position detection is unnecessary. Since one current sensor is enough for CPPM BSG with trapezoidal back EMF in measuring both phase current and DC bus current [5], only rotor position detection method in the starter state is discussed in this paper.

Hall sensors have the benefits of low cost, simple signal processing, vibration resistance and dust proof [6] in contrast to resolver and quadrature encoder which are mainly used to detect rotor position in CPPM machine [7]. As six-step commutation requires a 60 electrical degrees resolution in rotor position signal, the precision of Hall sensors is sufficient.

In a BLDC motor, three Hall sensors are mounted with 120 electrical degrees phase shift to each other [4]. However, rotor position detection methods in BLDC motor cannot be used in CPPM BSG due to the asymmetric flux distribution in each pole side since wrong rotor position signals can lead to unbalanced motor operation or even failure in six-step commutation.

To simplify the control of CPPM BSG with trapezoidal back EMF and thus reduce the cost of sensors, a new rotor position detection method using six Hall sensors is proposed in this paper in which six Hall sensors position and signal processing are discussed based on the analysis 
of magnetic field characteristic in each pole side. Experiments of a $2 \mathrm{~kW}$ CPPM BSG prototype have also been performed to verify the proposed method.

\section{Magnetic Field Analysis}

The structure of CPPM BSG is shown in Fig. 1 in which both the stator and rotor are divided into North and South pole side and the rotor in each pole side is partially surface-mounted PMs while the other part is iron. Each pole is composed of a PM and iron from different pole sides and a field winding is placed between armature cores of North and South pole side. CPPM BSG works with the demagnetizing or magnetizing effect when the direction of the field current is adjusted [5]. When the direction of the field current is clockwise, the direction of the air gap flux in one pole is the same. As the result, the total air gap flux per pole increases as the flux current increases, making CPPM BSG work with the magnetizing effect. On the other hand, when the direction of the field current reverses, CPPM BSG would work in the demagnetizing effect.

In the starter state, field current is fixed at the maximum value of magnetizing effect. The $2 \mathrm{D}$ structure and magnetic paths of CPPM BSG is shown in Fig. 2, where $h_{1}$ is the width of $\mathrm{PM}, h_{2}$ is the height of iron and $\delta$ is the length of air gap. Magnetic path of PM flux is shown in Fig. 2(a) in which the main PM flux flows from the PM pole of the North pole side to the South pole side through the stator and rotor yoke. The main field flux flows from the iron pole of South pole side to North pole side through the stator and rotor yoke, as depicted in Fig. 2(b). Moreover, there is flux in the region between PM and iron whose direction can change the proportion of North and South pole magnetic field in each pole side.

The magnetic equivalent circuit of flux in the region between PM and iron is depicted in Fig. 3 in which the flux generated by PM and field magnetomotive force

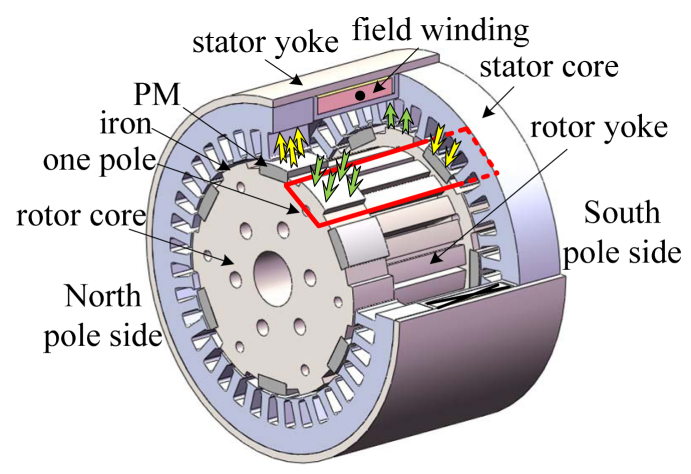

Fig. 1. (Color online) Structure of CPPM BSG.

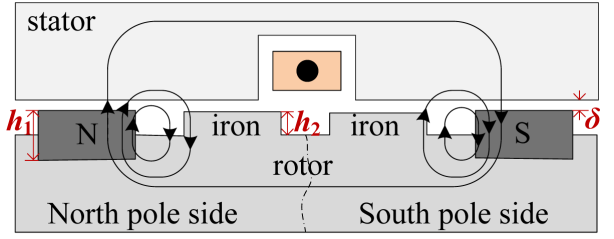

(a)

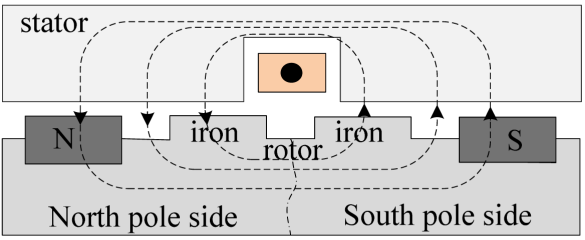

(b)

Fig. 2. (Color online) Magnetic path of CPPM BSG. (a) Magnetic path of PM flux. (b) Magnetic path of field flux.

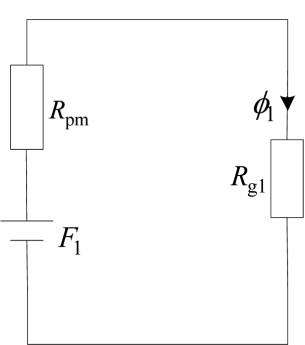

(a)

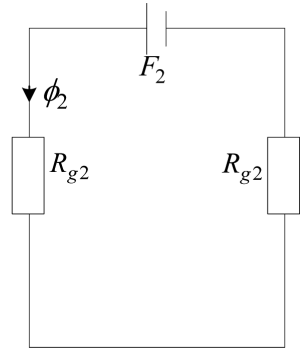

(b)
Fig. 3. Magnetic equivalent circuit of flux in region between PM and iron. (a) Path of PM flux. (b) Path of field flux.

(MMF) in the region between PM and iron can be expressed as:

$$
\begin{aligned}
& \Phi_{1}=F_{1} /\left(R_{\mathrm{g} 1}+R_{\mathrm{pm}}\right) \\
& \Phi_{2}=F_{2} /\left(2 R_{\mathrm{g} 2}\right)
\end{aligned}
$$

where $\Phi_{1}, \Phi_{2}$ are the flux generated by PM and field MMF in the region between PM and iron, respectively; $F_{1}, F_{2}$ are $\mathrm{PM}$ and field $\mathrm{MMF}$ and $R_{\mathrm{pm}}, R_{\mathrm{g} 1}, R_{\mathrm{g} 2}$ are $\mathrm{PM}$ reluctance, air gap reluctance in magnetic path for PM and field flux in the region between PM and iron, respectively.

In this application, $\Phi_{1}$ and $\Phi_{2}$ have a different sign, $\left|F_{2}\right| \approx\left|2 F_{1}\right|$ and $h_{1} \geq h_{2}>\delta$. The length of air gap in magnetic path of PM flux in the region between PM and iron cannot be lower than $h_{2}$ and the air gap of magnetic path of field flux in the region between PM and iron is $h_{2}+\delta$. Besides, the permeability of PM and air is almost the same, which can be deduced as $\left(h_{1}+h_{2}\right)>\left(h_{2}+\delta\right)$. Since the reluctance is determined by permeability and length of the medium, $\left(R_{\mathrm{g} 1}+R_{\mathrm{pm}}\right)>R_{\mathrm{g} 2}$. Furthermore, 


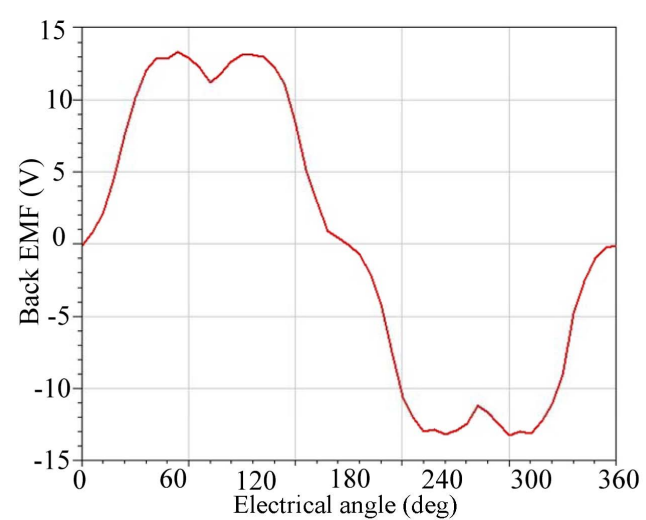

(a)

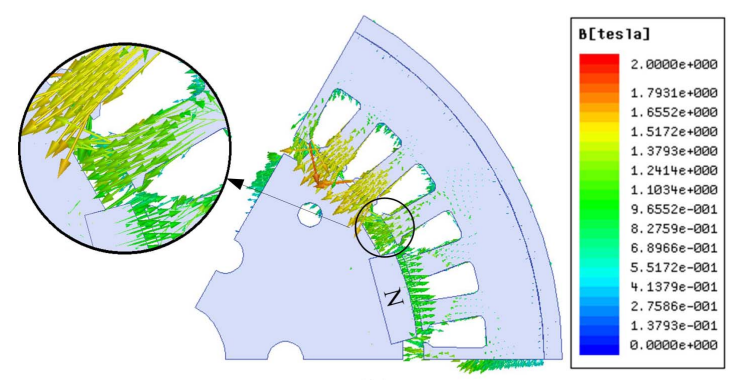

(b)

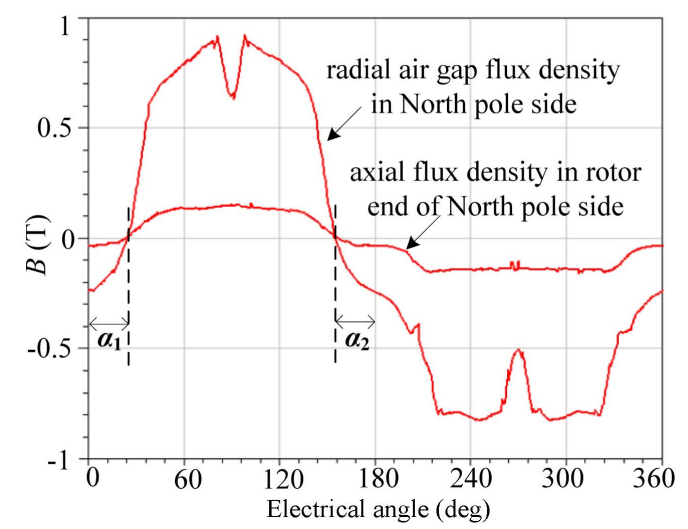

(c)

Fig. 4. (Color online) 3D FEA results of CPPM BSG. (a) Back EMF waveform. (b) Flux density vector of North pole side. (c) Flux density distribution along circumference of North pole side.

since $\Phi_{2}$ and $\Phi_{2}$ are calculated using (1) and (2), it is obvious that $\left|\Phi_{2}\right|<\left|\Phi_{2}\right|$. Therefore, flux in the region between PM and iron and flux in iron has the same direction, which means that the proportion of North and South pole magnetic field in each pole side is unequal.

Parameters of the prototype are: $h_{1}=5 \mathrm{~mm}, h_{2}=2 \mathrm{~mm}$, $\delta=1 \mathrm{~mm}$ and pole pairs $2 p=6$. The flux distribution of prototype analyzed by 3D FEA is shown in Fig. 4, in which the positive value means that the direction of the flux is from North pole to South pole.

The back EMF waveform of prototype is trapezoidal and symmetric, as shown in Fig. 4(a), which indicates that the synthetic radial air gap flux density waveform is trapezoidal and passes through zero at $0^{\circ}$ and $180^{\circ}$. In each pole side, however, the direction of the flux in the region between PM and iron is the same as that in iron, as shown in Fig. 4(b). The positive part of radial air gap flux density in North pole side is wider in an electric angle cycle and the angle deviations of zero crossing points between radial air gap flux density in North pole side and synthetic radial air gap flux density are $\alpha_{1}$ and $\alpha_{2}$, as shown in Fig. 4(c). The axial flux density distribution in the rotor end is along circumference and about $2 \mathrm{~mm}$ apart from the rotor end in axial direction. As shown in Fig. 4(c), due to the dispersivity of magnetic field, the axial flux density in rotor end and radial air gap flux density in North pole side pass through zero at the same points, which means that the same Hall signal can be obtained when a Hall sensor is used to sense axial flux density in rotor end and radial air gap flux density in the same pole side. Our simulation results will demonstrate that flux distribution of CPPM BSG is asymmetric in each pole side.

\section{Rotor Position Detection of CPPM BSG}

In BLDC motor, three Hall sensors are used to detect the rotor position. For each Hall signal, half of the electrical angle cycle is high level as North pole is passing near the sensor and the other half is low level as South pole is passing. The relationship between Hall signal and back EMF is discussed in [4], as shown in Fig. 5.

In CPPM BSG, as mentioned above, the flux density distribution is asymmetric in each pole side. If three Hall sensors are used in each pole side as in BLDC motor,

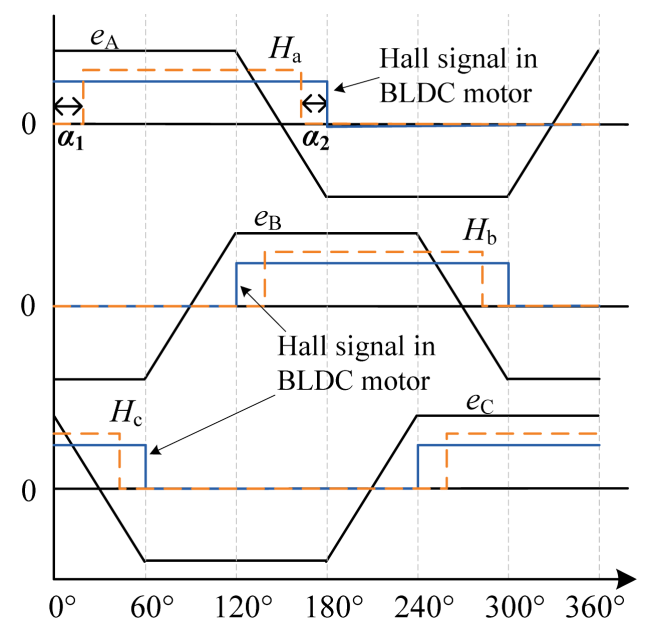

Fig. 5. (Color online) Signals of three Hall sensors with respect to back EMFs. 
there will be offset angles between the back EMF and the related Hall signal for the unequal angular duration of high and low level. Three Hall signals in North pole side with respect to back EMF are shown in Fig. 5, where $e_{\mathrm{x}}$ $(\mathrm{x}=\mathrm{A}, \mathrm{B}, \mathrm{C})$ are the back EMFs and $H_{\mathrm{a}}, H_{\mathrm{b}}, H_{\mathrm{c}}$ are signals of the three Hall sensors in CPPM BSG. With the conducting sequence of six-step commutation control strategy, the performance of CPPM BSG will be affected if the asymmetric Hall signals are used. To control sixstep commutation of CPPM BSG correctly, six Hall sensors are hence used to sense the rotor position.

The six Hall sensors are divided into three groups that correspond to the three phase windings. Group $\mathrm{x}(\mathrm{x}=\mathrm{A}$, $\mathrm{B}, \mathrm{C})$ consists of sensors $\mathrm{x} 1$ and $\mathrm{x} 2$ with the output signals being $H_{\mathrm{x} 1}$ and $H_{\mathrm{x} 2}$, respectively. The two signals in each group are combined through performing proper logic calculation. And the angular duration of high and low level of the combined signals can be changed by moving the Hall sensors in each group. Therefore, the offset angles $\alpha_{1}$ and $\alpha_{2}$ can be compensated by moving the two Hall sensors in each group.

Assume that Hall sensors are mounted in North pole side, and Hall signal is high level as North pole is passing near the sensor. For group A in Fig. 6, Hall sensor A1 is moved to the position where the rising edge of signal $H_{\mathrm{A} 1}$ is at 0 electrical degrees, Hall sensor A2 is moved to the position where the falling edge of signal $H_{\mathrm{A} 2}$ signal is at 180 electrical degrees. Then, by performing OR operation, signal $H_{\mathrm{A} 1}$ and signal $H_{\mathrm{A} 2}$ are combined into signal $H_{\mathrm{A}}$. Moreover, angle $\alpha\left(\alpha=\alpha_{1}+\alpha_{2}\right)$ cannot be more than 90 electrical degrees. Otherwise, signal $H_{\mathrm{A}}$ will become asymmetric in an electric angle cycle.

When Hall signal is at low level as North pole is passing near the sensor, signal $H_{\mathrm{A} 1}$ and signal $H_{\mathrm{A} 2}$ should perform NAND operation for the following reason.

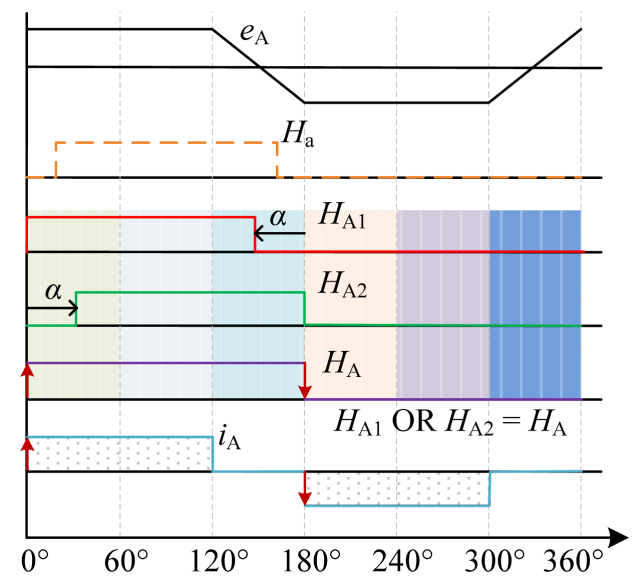

Fig. 6. (Color online) Logic calculation of Hall signals in group A.

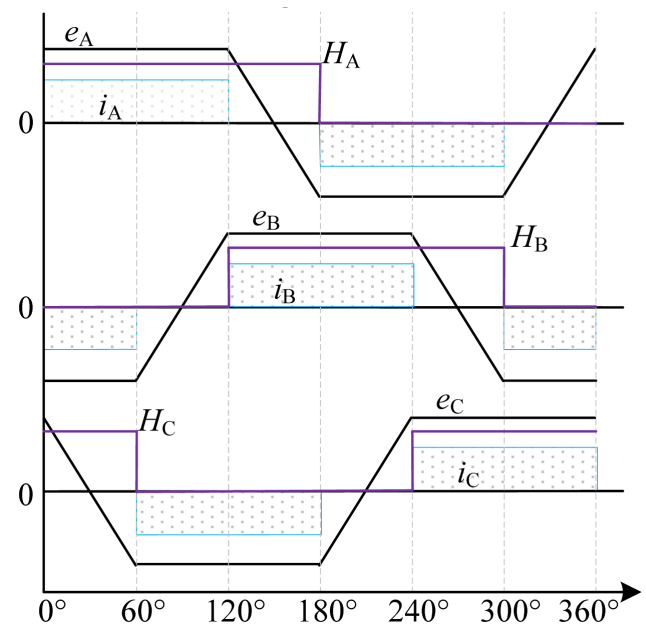

Fig. 7. (Color online) Processed signals with respect to back EMFs and phase currents.

$$
H_{\mathrm{A} 1}+H_{\mathrm{A} 2}=\overline{\overline{H_{\mathrm{A} 1}}} \bullet \overline{\overline{H_{\mathrm{A} 2}}}
$$

As Hall sensors of group B and group $\mathrm{C}$ lag behind the Hall sensors of group A in turn with 120 electrical degrees, the processed signals of group $\mathrm{B}$ and group $\mathrm{C}$ shown in Fig. 7 can be deduced easily, where $i_{\mathrm{x}}(\mathrm{x}=\mathrm{A}$, $\mathrm{B}, \mathrm{C})$ are the phase currents, $H_{\mathrm{A}}, H_{\mathrm{B}}, H_{\mathrm{C}}$ are the processed signals of groups $\mathrm{A}, \mathrm{B}$ and $\mathrm{C}$, respectively. The rising edges of signals $H_{\mathrm{A}}, H_{\mathrm{B}}, H_{\mathrm{C}}$ fall behind the back EMF zero crossing point of phases $\mathrm{A}, \mathrm{B}$ and $\mathrm{C}$, respectively, and all the lagging angles are 30 electrical degrees. Signals $H_{\mathrm{A}}, H_{\mathrm{B}}, H_{\mathrm{C}}$ are the same as the Hall signals of BLDC motor in Fig. 5.

\section{Experimental Results}

The circuit of six Hall sensors is shown in Fig. 8(a), where $R$ is pull-up resistors. Hall sensors A1, B1, C1 and A2, B2, C2 are placed 120 electrical degrees apart, respectively. The six Hall sensors along with the related circuit and logic chip can be integrated into a printed circuit board (PCB) inside CPPM BSG, the two Hall signals in each group perform proper operation separately, and processed signals $H_{\mathrm{A}}, H_{\mathrm{B}}, H_{\mathrm{C}}$ from the logic chip are connected to the controller. As the result, only three signal wires are necessary between CPPM BSG and controller. Moreover, the six Hall sensors and one logic chip are cheap enough, making the rotor position detection method cost effective.

The rotor of CPPM BSG is divided into two parts by rotor yoke and the poles are composed of PM and iron, as shown in Fig. 8(b). The PCB is fixed on the end bracket next to North pole side and the model of the Hall sensors is US1881 as shown in Fig. 8(c). The marker side of the 


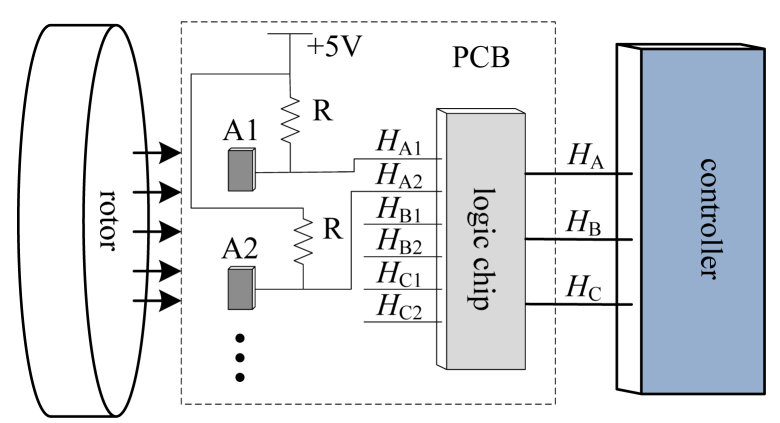

(a)

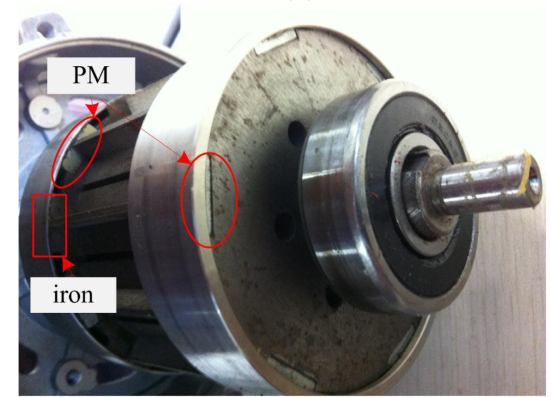

(b)

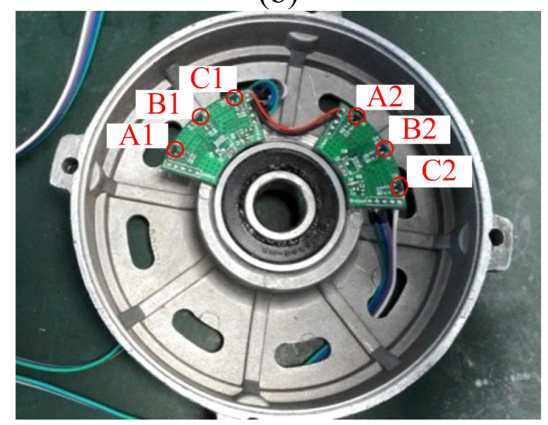

(c)

Fig. 8. (Color online) Circuit of Hall sensors and installation. (a) Circuit of Hall sensors. (b) Prototype rotor. (c) End bracket with PCB.

Hall sensor faces to the rotor and senses the axial flux density in prototype rotor end. The Hall signal switches to the high level when the marker side faces to the magnetic South pole. Therefore, the two Hall signals in each group would perform NAND operation separately in the experiment.

The experiment platform is shown in Fig. 9, which consists of a controller, a CPPM BSG, a magnetic powder brake, an induction motor and a torque sensor and apparatus. To test the Hall signal conveniently, the logic chip is placed outside of the CPPM BSG. Hall signals and back EMF can be measured when CPPM BSG is driven by induction motor. In addition, CPPM BSG is powered by controller in starting test. The test results are shown in Fig. 10.

To control six-step commutation in the right sequence,

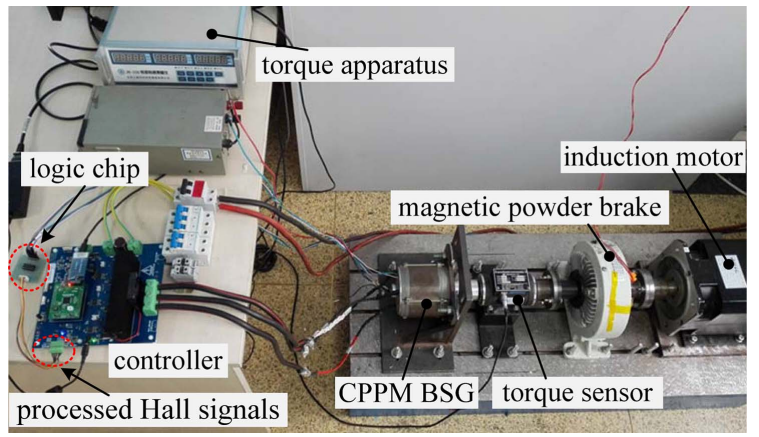

Fig. 9. (Color online) Experimental platform.

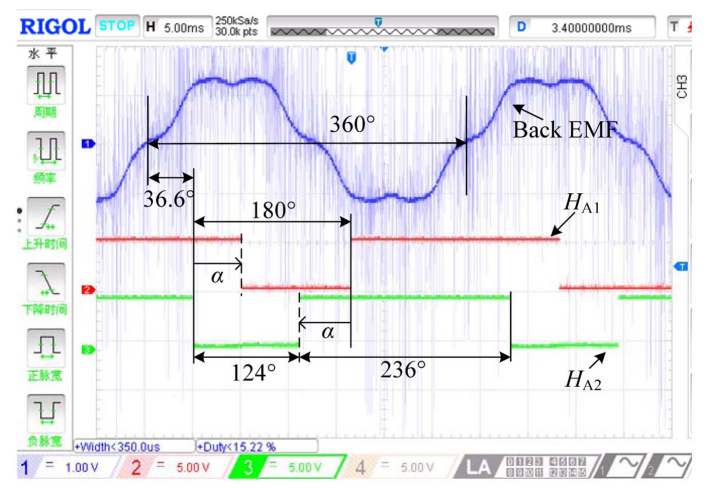

(a)

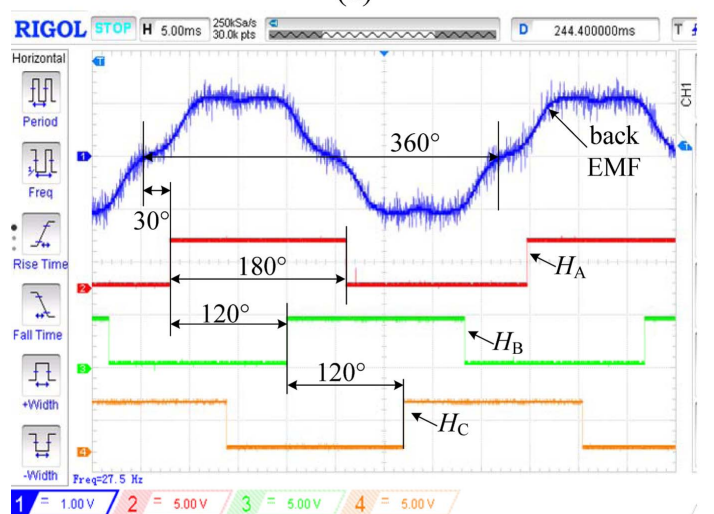

(b)

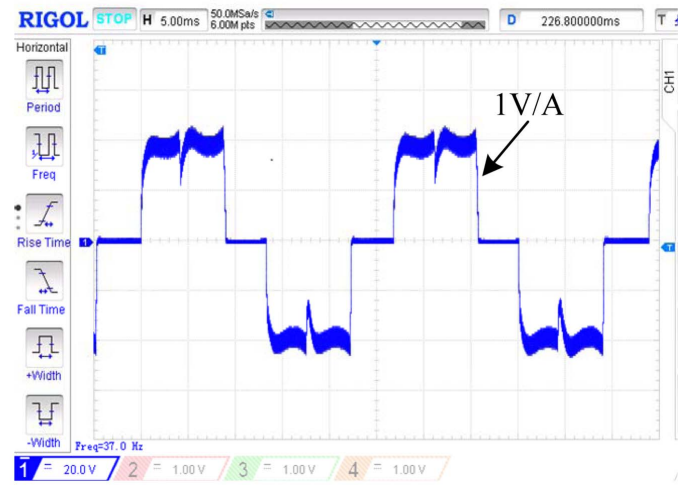

(c)

Fig. 10. (Color online) Test results. (a) Two signals of group $\mathrm{A}$ and back EMF. (b) The processed Hall signals and back EMF. (c) Phase current in starting test. 
the lagging angle between rising edge of signal $H_{\mathrm{A} 1}$ and zero crossing point of back EMF should be 30 electrical degrees, which can be achieved by moving the PCB along circumference. For instance, Fig. 10(a) shows two signals of group A and back EMF of phase A when PCB is mounted in the wrong place. From signals $H_{\mathrm{A} 1}$ and $H_{\mathrm{A} 2}$, it can be deduced that the angular duration of North pole magnetic field is 124 electrical degrees and angle $\alpha$ is 56 electrical degrees, which satisfies the restricted condition mentioned above. However, the lagging angle between rising edge of signal $H_{\mathrm{A} 1}$ and the zero crossing point of back EMF is 36.6 electrical degrees. Therefore, the PCB should be moved forward with 6.6 electrical degrees.

Figure 10 (b) shows processed signals $H_{\mathrm{A}}, H_{\mathrm{B}}, H_{\mathrm{C}}$ with respect to back EMF of phase A after the PCB is adjusted to proper place and the two Hall signals from each group perform NAND operation. The rising edges of signals $H_{\mathrm{A}}$, $H_{\mathrm{B}}, H_{\mathrm{C}}$ fall behind the back EMF zero crossing point of phases $\mathrm{A}, \mathrm{B}$ and $\mathrm{C}$, respectively, and all the lagging angles are 30 electrical degrees. The angular duration of high level is 180 electrical degrees in an electric angle cycle. Moreover, processed signals $H_{\mathrm{A}}, H_{\mathrm{B}}, H_{\mathrm{C}}$ is at 120 electrical degrees phase shift to each other.

Based on the rotor position signals of $H_{\mathrm{A}}, H_{\mathrm{B}}, H_{\mathrm{C}}$, sixstep commutation control is adopted in starting test, and field current is fixed at 7A to maximize the magnetizing effect. The phase current waveform in steady state is shown in Fig. 10(c), and the amplitude is 40 A. It is obvious that phase current is distributed uniformly, which is similar to that produced by BLDC motor.

Results of the experiment indicate that both the hall signals and phase current are similar between CPPM BSG with trapezoidal back EMF and BLDC motor. Furthermore, resolution of the six Hall sensors is enough for
CPPM BSG to perform the six-step commutation control.

\section{Conclusions}

CPPM BSG with trapezoidal back EMF can be regarded as BLDC motor in the starter state, thus six-step commutation control widely used in BLDC motor can be adopted. The magnetic field in each pole side of CPPM BSG is asymmetric and there are offset angles between back EMF and the related Hall signal, making it unsuitable to use rotor position detection methods with three Hall sensors in BLDC motor. However, by dividing six Hall sensors into three groups and moving the two Hall sensors in each group, the offset angle can be compensated. Furthermore, the processed signals which are produced by combining the two signals in each group can offer the correct rotor position for the six-step commutation control.

\section{References}

[1] Feng Chi Hsieh, Yin Dar Huang, in Automation Science and Engineering, IEEE Int. Conf. (2014) pp. 916-921, 1822.

[2] T. Mizuno, K. Nagayama, and T. Ashikaga, Electrical Engineering in Japan 117, 5 (1996).

[3] J. A. Tapia, F. Leonardi, and T. A. Lipo, IEEE Trans. Ind. Appl. 39, 6 (2003).

[4] P. Pillay and R. Krishnan, IEEE Trans. Ind. Appl. 25, 2 (2003).

[5] S. D. Sudhoff and P. C. Krause, IEEE Trans. Energy Convers. 5, 3 (1990).

[6] Wang Cheng, Deng Zhi-quan, and Cai Jun, Small \& Special Electrical Machines 41, 3 (2014).

[7] Zheran Li, Yesong Li, and Xinhua Li, IEEE Trans. Power Electron. 29, 9 (2014). 